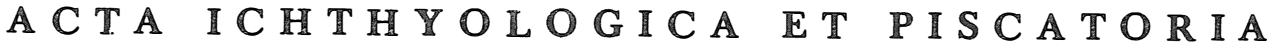 Vol. IX Fasc. 1

T. VENKATESWARLU and A.G.K. MENON

Systematies

\section{A LIST OF FISHES OF THE RIVER GANGES AND ITS BRANCHES}

\section{WYKAZ RYB RZEKI GANGES I JEJ DOPLYWÓW}

Zoological Survey of India, Southern Regional Station-Madras

The authors give a taxonomic check list 264 fish species occurring in the Ganges and compare the newly - compiled list with previous ones by Hamilton and Day.

\section{INTRODUCTION}

Since Hamilton-Buchanan's account (1822) of fishes found in the river Ganges and its branches published no other comprehensive account has been published. Day (1875-1888), Chaudhuri (1912), Hora (1921, 1929, 1938, 1948, 1949, 1955), Hora and Mukheree(1936), Shaw and Shebbeare (1938), Chauhan (1947), Das (1948), Menon (1949, 1949a, 1962, 1971 and 1974), Jayaram (1953, 1962), Sinha and Shiromony (1953), David (1954), Jhingran (1956), Motwani and David (1957), Majumdar (1958), Misra (1959), Lal and Chatterjee (1962), Karamchandani (1962), Mahajan (1965), Pant (1966), Srivastava $(1967,1968)$ and Venkateswarlu (1972, 1973, 1976 and 1977), published some papers dealing with Ichthyofauna of this river system. Hamilton's work contains descriptions of 271 species, while one species is mentioned only by name (p. 316). He figured 198 fishes in 39 plates on the Atlas accompanying the text; 5 of them on plates $\mathrm{ii}, \mathrm{iv}, \mathrm{xii}, \mathrm{xxxiii}$ and $\mathrm{xxxv}$ are drawings of special parts of fish. 93 are outline sketches shiwing the fish from above and 100 finished drawings of fish in lateral view. Of 
the 100 drawings two are to illustrate the juvenile forms of the species figured also as adults (pls. xxxiv and xvii). The grawings of Ophisurus hijala (pl.v, fig. 5) belongs to an underscribed species which Hamilton called Muraena serpens in his original notes ${ }^{1}$ and he evidently mixed up the drawings and descriptions of the two species when publishing. Thus it is clear that of the total of 271 species described in the "Gangetic Fishes" delineations of only 97 species are published in his work. Hamilton, however, left behind him in India original drawings of 144 species of fish which are now preserved in the Asiatic Society of Bengal. These drawings are of great importance in that they formed the basis of the descriptions of the fishes in the "Gangetic fishes" (Hora, 1929) and especially since left no type specimens.

Of the 272 species described in the "Gangetic Fishes", 264 species are included in the present check list, the systematic position of eight of the following species being uncertain: 1. Muraena vomos 2. Clupanodon chanpole, 3. Clupanodon cagius, 4. Cyprinus (Puntius) joalius, 5.Cyprinus (Cabdio) hoalius, 6. Cyprinus (Cabdio) solio, 7. Cyprinus (Garra) mosario, 8. Bola coibor.

The classification followed is after Berg (1940). The genera and species are arranged in alphabetical order. Against each species whose current scientific name is given in the first column, the names as rendered in the "Fishes of India" by Day (1875-1888) and Hamilton in his "Gangetic Fishes" are given in the next two columns respectively.

1 There are three volumens of Hamilton-Buchanan's manuscripts in the Indian Office Library in London. One of these contains the original notes on Gangetic Fishes and the othes two formed the Manuscript of his published work. 


\section{LIST OF FISHES}

\begin{tabular}{|c|c|c|}
\hline Current scientific Name & $\begin{array}{l}\text { Name as given in Day's } \\
\text { Fish India }\end{array}$ & $\begin{array}{c}\text { Name as given in Hamilton's } \\
\text { Gangetic Fishes }\end{array}$ \\
\hline 1 & 2 & 3 \\
\hline $\begin{array}{l}\text { Class: } \\
\text { Sub Class: } \\
\text { Order: } \\
\text { Sub Order: } \\
\text { Family: }\end{array}$ & $\begin{array}{l}\text { ELASMOBRANCHII } \\
\text { SELACHII } \\
\text { LAMNIFORMES } \\
\text { SCYLIORHINCHIDEI } \\
\text { Carcharinidae } \\
\text { Genus: Carcharinus Blainville }\end{array}$ & \\
\hline $\begin{array}{l}\text { Carcharinus gangeticus } \\
\text { (Müller et Henle) }\end{array}$ & $\begin{array}{l}\text { Carcharinus gangeticus } \\
\text { (Müller et Henle) } \\
\text { Genus: Hypoprion Müller et }\end{array}$ & $\begin{array}{l}\text { Squalus carcharias } \\
\text { Henle }\end{array}$ \\
\hline $\begin{array}{l}\text { Hypoprion hemiodon } \\
\text { (Müller et Henle) }\end{array}$ & Carcharias hypoprion & \\
\hline $\begin{array}{l}\text { Order: } \\
\text { Family: }\end{array}$ & $\begin{array}{l}\text { RAJIFORMES } \\
\text { Pristidae } \\
\text { Genus: Pristis Latham }\end{array}$ & \\
\hline $\begin{array}{l}\text { Pristis pectinatus } \\
\text { Latham }\end{array}$ & Pristis pectinatus & Squalus pectinatus \\
\hline Family: & $\begin{array}{l}\text { Trigonidae (Dasyatidae) } \\
\text { Genus: Dasyatus Rafinesque }\end{array}$ & \\
\hline $\begin{array}{l}\text { Dasyatus (Humantura) mar- } \\
\text { ginatus (Blyth) }\end{array}$ & Trygon $\overline{\text { marginatus }}$ & \\
\hline $\begin{array}{l}\text { Dasyatus (Pestinachus) } \\
\text { sephen (Forskal) }\end{array}$ & Trygon sephen & Raja fluviatilis \\
\hline $\begin{array}{l}\text { Class: } \\
\text { Sub Class: } \\
\text { Order: } \\
\text { Sub Order: }\end{array}$ & $\begin{array}{l}\text { TELEOSTOMI } \\
\text { ACTINOPTER YGII } \\
\text { CLUPEIFORMES } \\
\text { CLUPEOIDEI }\end{array}$ & \\
\hline Family: & $\begin{array}{l}\text { Megalopidae } \\
\text { Genus: Megalops Lacépède }\end{array}$ & \\
\hline $\begin{array}{l}\text { Megalops cyprinoides } \\
\text { (Brouss.) } \\
\text { Family: }\end{array}$ & $\begin{array}{l}\text { Megalops cyprinoides } \\
\text { Clupeidae } \\
\text { Genus: Anodontostoma Blee }\end{array}$ & Cyprinodon cundinga \\
\hline $\begin{array}{c}\text { Anodontostoma chacunda } \\
\text { (Ham.) }\end{array}$ & Chatoessus chacunda & $\begin{array}{l}\text { Clupanodon chacunda } \\
\text { Clupanoaion chanpole } \\
\text { Clupanodon cagius }\end{array}$ \\
\hline
\end{tabular}




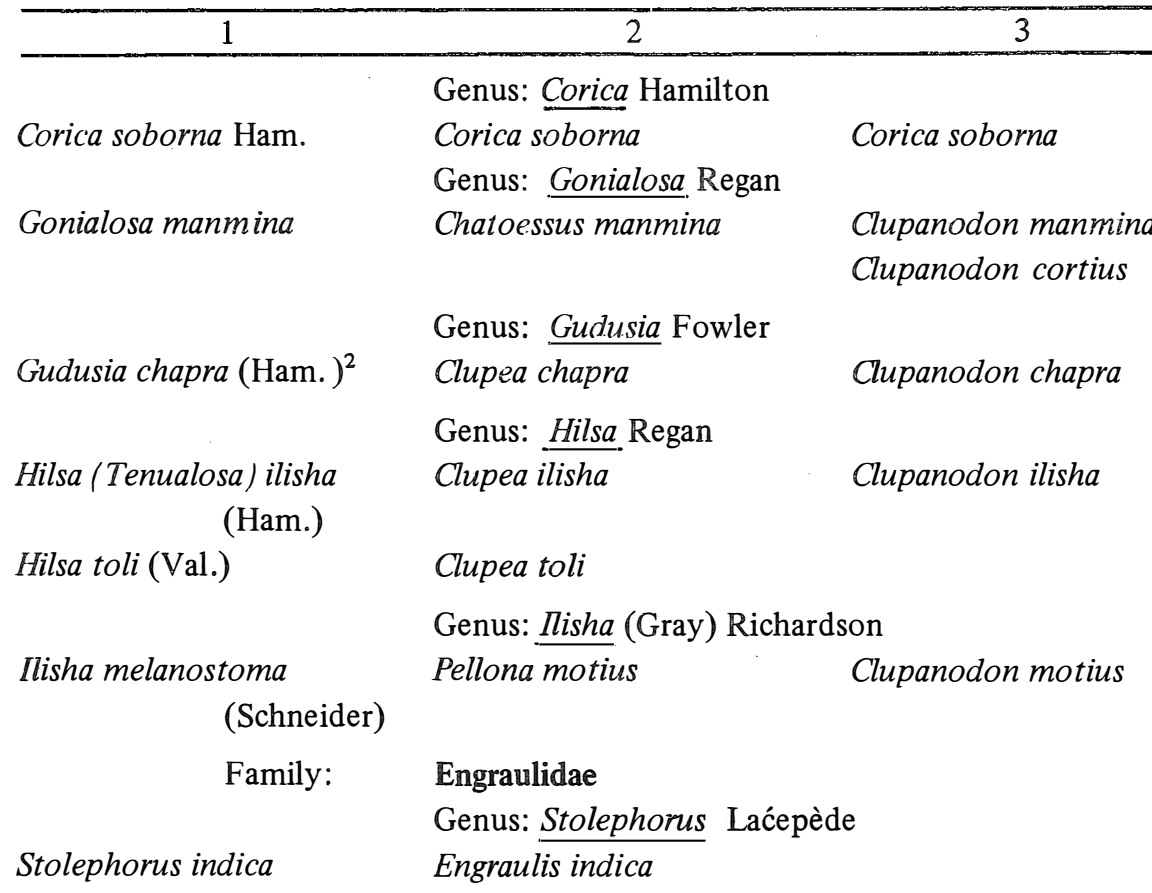

(V. Hass.)

$\begin{array}{ll} & \text { Setipinna phasa } \\ \text { Stolephorus tri (Blecker) } & \begin{array}{l}\text { Engraulis tri } \\ \text { Genus: Coilia Gray }\end{array} \\ & \text { Coilia dussumieri } \\ \text { Coilia dussumieri Val. } & \text { Coilia ramcarati } \\ \text { Coilia ramcarati (Ham.) } & \text { Coilia reynaldi } \\ \text { Coilia reynaldi } \text { Val. } & \end{array}$

Genus: Setipinna Swainson

Setipinna phasa (Ham.) Engraulis telera Clupea phasa

Genus: Thryssa Cuvier

Thryssa hamiltoni (Gray) Engraulis hamiltoni

Thryssa purava (Ham.) Engraulis purava Clupea purava

Sub Order: NOTOPERTOIDEI

Family: $\quad$ Notopteridae

Genus: Notopterus Laćepède

Notopterus chitala (Ham.) Notopterus chitala Mystus chitala

Notopterus notopterus Notopterus kapirat Mystus kapirat

(Pallas)

${ }^{2}$ Clupea suhia Chaudhuri and Gudusia godanahiai Srvastava are synonymous with Gudusia chapra (Ham.) (vide Whitehead, 1973) 


\begin{tabular}{cl}
\hline Order: & \multicolumn{1}{c}{3} \\
Family: & $\begin{array}{l}\text { SCOPELIFORMES } \\
\text { Synodidae } \\
\end{array}$ \\
& Genus: Harpodon Le Sue \\
Harpodon nehereus (Ham.) & Harpodon nehereus \\
Order: & CYPRINIFORMES \\
Sub Order: & CYPRINOIDEI \\
Family: & Cyprinidae \\
Sub Family: & Abramidinae \\
& Genus: Chela Hamilton
\end{tabular}

Chela cachius (Ham.) ${ }^{3} \quad$ Perilampus atpar

Osmerus nehereus

Chela laubuca (Ham.)

Perilampus atpar

Cyprinus (Chela) cachius

Cyprinus (Chela) atpar

Cyprinus (Cabdio) laubuca

Cyprinus (Cabdio) dancena

Genus: Oxygaster Van Hasselt

Oxygaster argentea (Day) Chela argentea

Oxygaster bacaila (Ham.) Chela bacaila

Cyprinus (Chela) baicala

Chela punjabensis

Oxygaster boopis (Day) Chela boopis

Oxygaster clupeoides Chela clupeoides

(Bloch)

Oxygaster gora (Ham.)

Chela gora

Cyprinus (Chela) gora

Oxygaster phulo (Ham.)

Oxygaster sardinella

Chela phulo

Cyprinus (Chela) phulo

(Cuv. et Val.)

Sub Family: Rasborinae

Barilius barila (Ham.) $)^{4}$

Barilius barna (Ham.)

Barilius bendelisis (Ham.) ${ }^{5}$
Genus: Barilius Hamilton

Barilius barila

Barilius barna

Barilius bendelisis
Cyprinus (Barilius) barila
Cyprinus (Barilius) chedrio
Cyprinus (Barilius) barna
Cyprinus (Barilius) bendelisis
Cyprinus (Barilius) chedra
Cyprinus (Barilius) cosca
Cyprinus (Barilius) tila
Cyprinus (Cabdio) borelio

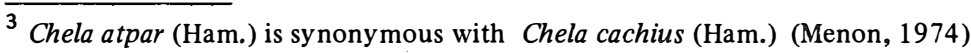

${ }^{4}$ Barilius bonarensis Chaudhuri is synonymous with B. barila (Ham.) (Menon, 1974)

${ }^{5}$ Barilius bendelisis var chedra (Ham.) is synonymous with Berilius bendelisis (Ham.) (Menon, 1974).
} 


\begin{tabular}{|c|c|}
\hline 1 & 2 \\
\hline Barilius bola (Ham.) & Barilius bola \\
\hline Barilius shacra (Ham.) & Barilius shacra \\
\hline Barilius tileo (Ham.) & Barilius tileo \\
\hline Barilius vagra (Ham.) & $\begin{array}{l}\text { Barilius vagra } \\
\text { Barilius modestus }\end{array}$ \\
\hline & Genus: Danio Hamilton \\
\hline $\begin{array}{r}\text { Danio (Danio) aequipinnatus } \\
\text { (Mc Clelland) }\end{array}$ & Danio aequipinnatus \\
\hline $\begin{array}{r}\text { Danio (Danio) dangila } \\
\text { (Ham.) }\end{array}$ & Danio dangila \\
\hline $\begin{array}{r}\text { Dania (Danio) devario } \\
\text { (Ham.) }\end{array}$ & Danio devario \\
\hline Danio (Brachydanio) rerio & Danio rerio \\
\hline & Genus: Esomus Swainson \\
\hline Esomus danricus (Ham.) & Nuria danrica \\
\hline
\end{tabular}

Cyprinus (Danio) dangila

Cyprinus (Babdio) devario

Cyprinus (Danio) rerio

Cyprinus (Danio) chapalio

Cyprinus (Barilius) bola

Cyprinus (Barilius) goha

Cyprinus (Barilius) shacra

Cyprinus (Barilius) tileo

Cyprinus (Barilius) vagra

Cyprinus (Danio) danrica

Cyprinus (Danio) suhita

Cyprinus (Danio) jogia

Genus: Rasbora Bleeker

Rasbora daniconius (Ham.) Rasbora daniconius

Cyprinus (Danio) daniconius

Cyprinus (Danio) anjana

Rasbora elanga (Ham.) Rasbora elanga

Cyprinus (Bangana) elanga

Rasbora rasbora (Ham.) Rasbora buchanani

Cyprinus (Danio) rasbora

Sub Family: Cyprininae

Acrossocheilus hexagono- Barbus hexagonolepis

lepis (Mc Clelland) Barbus dukai

Genus: Amblypharyngodon Bleeker

Amblypharyngodon micro- Amblypharyngodon micro-

lepis (Bleeker) lepis

Amblypharyngodon mola Amblypharyngodon mola Cyprinus (Cabdio) mola

(Ham.)

Genus: Aspidoparia Heckel

Aspidoparia jaya (Ham.) Aspidoparia jaya

Cyprinus (Cabdio) jaya

Aspidoparia morar (ham.) Aspidoparia morar

Cyprinus (Cabdio) morar

Genus: Catla Valenciennes

Catla catia (Ham.)

Catla buchanani

Cyprinus (Cyprinus) catla 
Genus: Chagunio Smith

Chagunio chagunio (Ham.) Barbus chagunio Cyprinus (Cyprinus) chagunio

Genus: Cirrhina (Oken) Cuvier

$\begin{array}{lll}\text { Cirrhina mrigala (Ham.) } & \text { Cirrhina mrigala } & \text { Cyprinus (Bangana) mrigala } \\ \text { Cirrhina reba (Ham.) } & \text { Cirrhina reba } & \text { Gyprinus (Bangana) reba }\end{array}$

Genus: Crossocheilus Van Hasselt

\begin{tabular}{|c|c|c|}
\hline $\begin{array}{c}\text { Crossocheilus latius } \\
\text { latius (Ham.) }\end{array}$ & Cirrhina latia & $\begin{array}{l}\text { Cyprinus (Garra) latius } \\
\text { Cyprinus (Garra) gohama } \\
\text { Cyprinus (Garra) sada }\end{array}$ \\
\hline
\end{tabular}

Garra annandalei Hora

Genus: Garra Hamilton

Garra gotyla gotyla

Discognathus lamta

(Gray)

Garra lamta (Ham.)

(in part)

Discognathus lamta

Cyprinus (Garra) lamta

(in part)

Genus: Labeo Cuvier

Labeo bata (Ham.)

Labeo bata

Cyprinus (Bangana) bata

Cyprinus (Bangana) acra

Cyprinus (Bangana) cura

Labeo boga (Ham.)

Labeo boga

Cyprinus (Bangana) boga

Labeo boggut (Sykes)

Labeo boggut

Labeo calbasu (Ham.)

Labeo calbasu

Cyprinus (Cyprinus) calbasu

Labeo dero (Ham.) $)^{6}$

Labeo microphthalmus

Cyprinus (Bangana) dero

Labeo diplostomus

Labeo sindensis

Labeo fimbriatus (Bloch)

Labeo fimbriatus

Cyprinus (Cyprinus) nancur

Labeo gonius (Ham.)

Labeo gonius

Cyprinus (Cyprinus) gonius

Cyprinus (Cyprinus) curchius

Cyprinus (Cyprinus) cursa

Cyprinus (Cyprinus) cursis

Labeo nandina (Ham.) Labeo nandina

Cyprinus (Cyprinus) nandina

Labeo pangusia (Ham.) $)^{7}$

Labeo pangusia

Cyprinus (Bangana) pangusia

Labeo angra

Cyprinus (Bangana) ariza

\footnotetext{
${ }^{6}$ Labeo almorhae Chaudhuri and Labeo rilli Chaudhuri are synonymous with Laboe dero (Ham.) (Menon, 1974).

7 Labeo kunki Chaudhuri and Labeo tezpurensis Chaudhuri are synonymous with Labeo pangusia (Ham.) (Menon, 1974). Cyprinus (Bangana) ariza recorded from the Ganges system by Hamilton is this species, Labeo ariza (Ham.) is found only in Nulgiris, Wynaad and Mysore.
} 


\begin{tabular}{|c|c|c|}
\hline 1 & 2 & 3 \\
\hline Labeo rohita (Ham.) & Labeo dyocheilus & $\begin{array}{l}\text { Cyprinus (Danio) angra } \\
\text { Cyprinus (Morulius! morala } \\
\text { Cyprinus (Morulius) pausius } \\
\text { Cyprinus (Morulius) musiha } \\
\text { Cyprinus (Puntius) pausio } \\
\text { Cyprinus (Cyprinus) rohita }\end{array}$ \\
\hline & Genus: Oreichthys Smith & \\
\hline Oreichthys cosuatis (Ham.) & $\begin{array}{l}\text { Barbus casuatis } \\
\text { Barbus apogon }\end{array}$ & Cyprinus (Cabdio) casuatis \\
\hline Osteobrama cotio (Ham.) & $\begin{array}{l}\text { Genus: Osteobrama Heckel } \\
\text { Rohtee }\end{array}$ & Cyprinus (Cabdio) cotio \\
\hline & Genus: Puntius Hamilton & \\
\hline Puntius ambassis (Day) & Barbus ambassis & \\
\hline Puntius amphibia (Val.) & Barbus amphibia & \\
\hline $\begin{array}{l}\text { Puntius chilinoides } \\
\text { (Mc Clelland) }\end{array}$ & Barbus chilinoides & \\
\hline Puntius chola (Ham.) & $\begin{array}{l}\text { Barbus chola } \\
\text { Barbus tetrarupagus }\end{array}$ & $\begin{array}{l}\text { Cyprinus (Puntius) chola } \\
\text { Cyprinus (Puntius) tictis } \\
\text { Cyprinus (Puntius) titius }\end{array}$ \\
\hline $\begin{array}{l}\text { Puntius elavatus } \\
\text { (Mc Clelland) }\end{array}$ & Barbus clavatus & \\
\hline Puntius conchonius & Barbus conchonius & Cyprinus (Puntius) conchonius \\
\hline Puntius curmuca (Ham.) & Barbus curmuca & Cyprinus (Cyprinus) curmuca \\
\hline Puntius gelius (Ham.) & Barbus gelius & $\begin{array}{l}\text { Cyprinus (Puntius) gelius } \\
\text { Cyprinus (Puntius) canius }\end{array}$ \\
\hline Puntius guganio (Ham.) & Barbus guganio & Cyprinus (Cyprinus) guganio \\
\hline Puntius phutunio (Ham.) & Barbus phutunio & Cyprinus (Puntius) phutunio \\
\hline Puntius punjabensis (Day) & Barbus punjabensis & \\
\hline $\begin{array}{l}\text { Puntius sarana sarana } \\
\qquad \text { (Ham.) }\end{array}$ & $\begin{array}{l}\text { Barbus sarana } \\
\text { Barbus chrysopoma } \\
\text { Barbus pinnauratus }\end{array}$ & Cyprinus (Cyprinus) sarana \\
\hline Puntius sophore (Ham.) & $\begin{array}{l}\text { Barbus sophore } \\
\text { Barbus stigma } \\
\text { Barbus puntio } \\
\text { Barbus chrysopterus }\end{array}$ & $\begin{array}{l}\text { Cyprinus (Puntius) sophore } \\
\text { Cyprinus (Puntius) puntio }\end{array}$ \\
\hline Puntius terio (Ham.) & Barbus terio & Cyprinus (Puntius) terio \\
\hline Puntius ticto (Ham.) & $\begin{array}{l}\text { Barbus ticto } \\
\text { Barbus stoliczkanus } \\
\text { Barbus punctatus }\end{array}$ & Cyprinus (Puntius) ticto \\
\hline
\end{tabular}


1

Semiplotus semiplotus
(Mc Clelland)
Tor putitora (Ham.)
Tor tor (Ham.)
Sub family:
Schizothorax kumaonensis
Menon

Schizothorax richardsoni

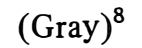

Schizothoraichthys pro-

gastus (Mc Clelland)

Family:

Genus: Tor Gray

Genus: Semiplotus Bleeker

Semiplotus macclellandi

Barbus tor (in part)

Barbus tor (in part)

Barbus hexasticus

Cyprinus (Cyprinus) putitora

Cyprinus (Cyprinus) tor

Schizothoracinae Heckel

Genus: Schizothorax Heckel

3

Oreinus richardsoni

Oreinus plagiostomus

Oreinus sinuatus

Genus: Schizothoraichtys Misra

Schizothorax progastus

Psilorhynchidae

Genus: Psilorhynchus Mc Clelland

Psilorhynchus balitora

Psilorhynchus balitora

Cyprinus (Garra) balitora

(Ham.)

Psilorhynchus pseudecheneis

Menon et Dutta

Psilorhynchus sucatio

Homaloptera bilineata

Cyprinus (Garra) sucatio

(Ham.)

Family: Homalopteridae

Baltitora brucei Gray

Genus: Baltitora Gray

Baltitora maculata Gray

Homaloptera brucei

Homaloptera maculata

Family: Cobitidae

$\begin{array}{cl}\text { Acanthophthalmus pangia } & \text { Genus: Acanthophthalmus } \\ \text { (Ham.) } & \text { Acanthophthalmus pangia Hasselt } \\ \text { Apua fusca }\end{array}$

${ }^{8}$ Diptychus annandalei Regan and Oreinus molesworthi Chaudhuri are synonymous with Schizothorax richardsoni (Gray), (Menon, 1974). 


\begin{tabular}{|c|c|c|}
\hline 1 & 2 & 3 \\
\hline & Genus: Botia Gray & \\
\hline Botia almorhae Gray & Botia almorhae & \\
\hline Botia dario (Ham.) & Botia dario & Cobitis dario \\
\hline Botia lohachata & Botia geto (in part) & Cobitis geto \\
\hline \multicolumn{3}{|l|}{ Chaudhuri } \\
\hline Botia rostrata Günther ${ }^{9}$ & \multicolumn{2}{|l|}{ Botia geto (in part) } \\
\hline $\begin{array}{c}\text { Lepidocephalus guntes }^{10} \\
\text { (Ham.) }\end{array}$ & $\begin{array}{l}\text { Genus: } \frac{\text { Lepidocephalus }}{B} \\
\text { Lepidocephalichthys } \\
\text { gunteha }\end{array}$ & $\begin{array}{l}\text { ker } \\
\text { Cobitis guntea } \\
\text { Cobitis balagara }\end{array}$ \\
\hline $\begin{array}{r}\text { Noemacheilus beavani } \\
\text { Günther }\end{array}$ & \multicolumn{2}{|l|}{ Nemacheilus beavani } \\
\hline Noemacheilus botia (Ham.) $)^{1}$ & ${ }^{1}$ Nemacheilus botia & $\begin{array}{l}\text { Cobitis botia } \\
\text { Cobitis bilturio }\end{array}$ \\
\hline $\begin{array}{l}\text { Noemacheilus corica } \\
\text { (Ham.) }\end{array}$ & Nemacheilus corica & Cobitis corica \\
\hline Noemacheilus dayi Hora & & \\
\hline $\begin{array}{r}\text { Noemacheilus danisoni } \\
\text { (Day) }\end{array}$ & Nemacheilus denisoni & \\
\hline $\begin{array}{r}\text { Noemacheilus montanus } \\
\text { (Mc Clelland) }\end{array}$ & Nemacheilus montanus & \\
\hline $\begin{array}{l}\text { Noemacheilus multifas- } \\
\text { ciatus Day }\end{array}$ & $\begin{array}{c}\text { Nemacheilus multifascia- } \\
\text { tus }\end{array}$ & \\
\hline $\begin{array}{l}\text { Noemacheilus rupicola } \\
\text { (Mc Clelland) }\end{array}$ & Nemacheilus rupicola & \\
\hline $\begin{array}{r}\text { Noemacheilus savona } \\
\text { (Ham.) }\end{array}$ & Nemacheilus savona & Cobitis savona \\
\hline Noemacheilus turio (Ham.) & Nemacheilus turio & Cobitis turio \\
\hline $\begin{array}{l}\text { Noemacheilus zonatus } \\
\text { (Mc Clelland) }\end{array}$ & Nemacheilus zonatus & \\
\hline
\end{tabular}

Genus: Somileptes Bleeker

Somileptes gongota (Ham.) Somileptes gongota

Cobitis gongota

Cobitis cucura

\footnotetext{
${ }_{9}^{9}$ Botia dayi Hora is synonymous with Botia rostrata Günther, (Manon, 1974).

${ }^{10}$ Lepidocephalichthys annandalei Chaudhuri is synonymous with Lepidocephalus guntea (Ham.), (Menon, 1974).

11 Nemacheilus mackenziei Chaudhuri is synonymous with Noemacheilus botta (Ham.), (Menon, 1974).
} 


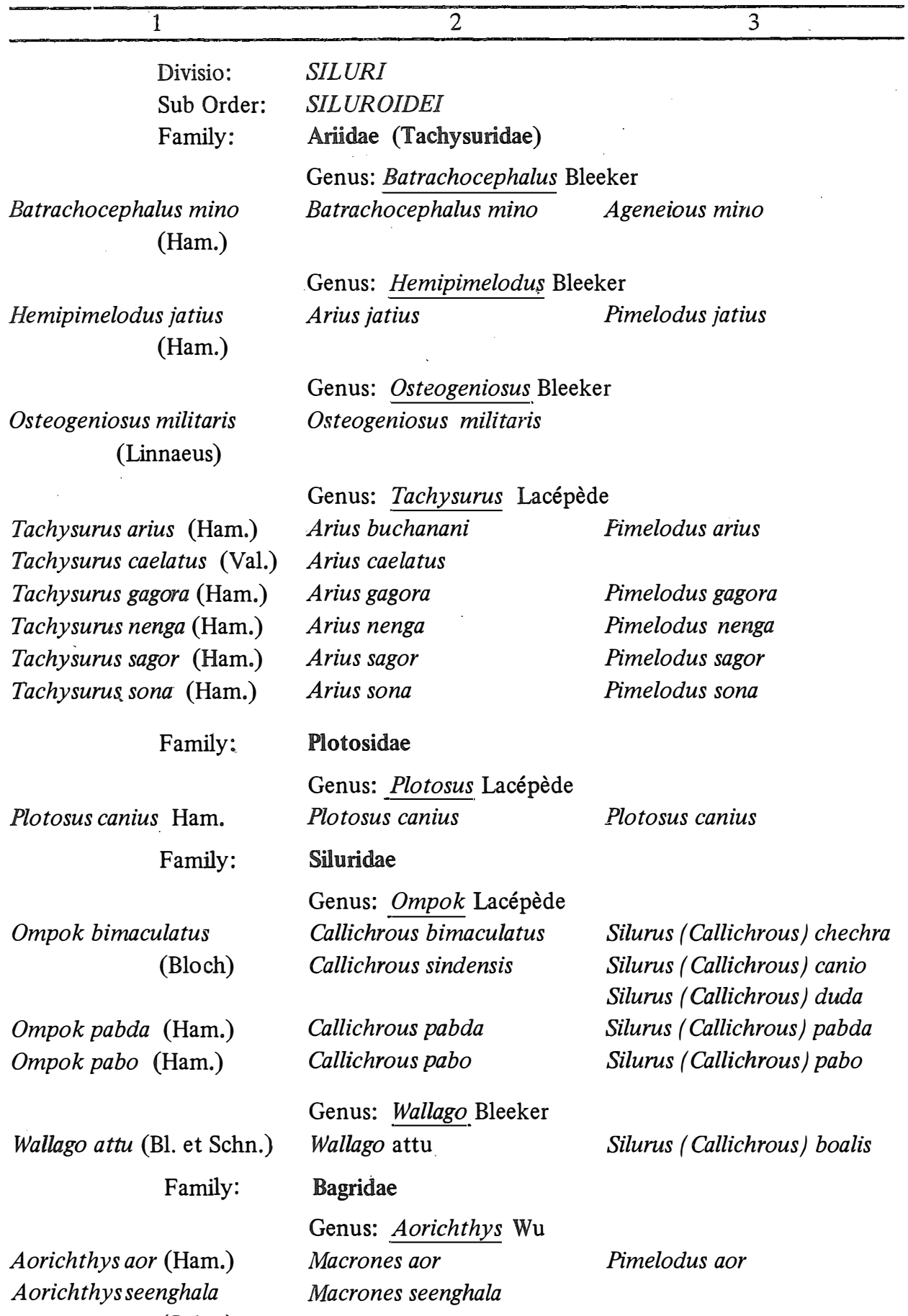

(Sykes) 


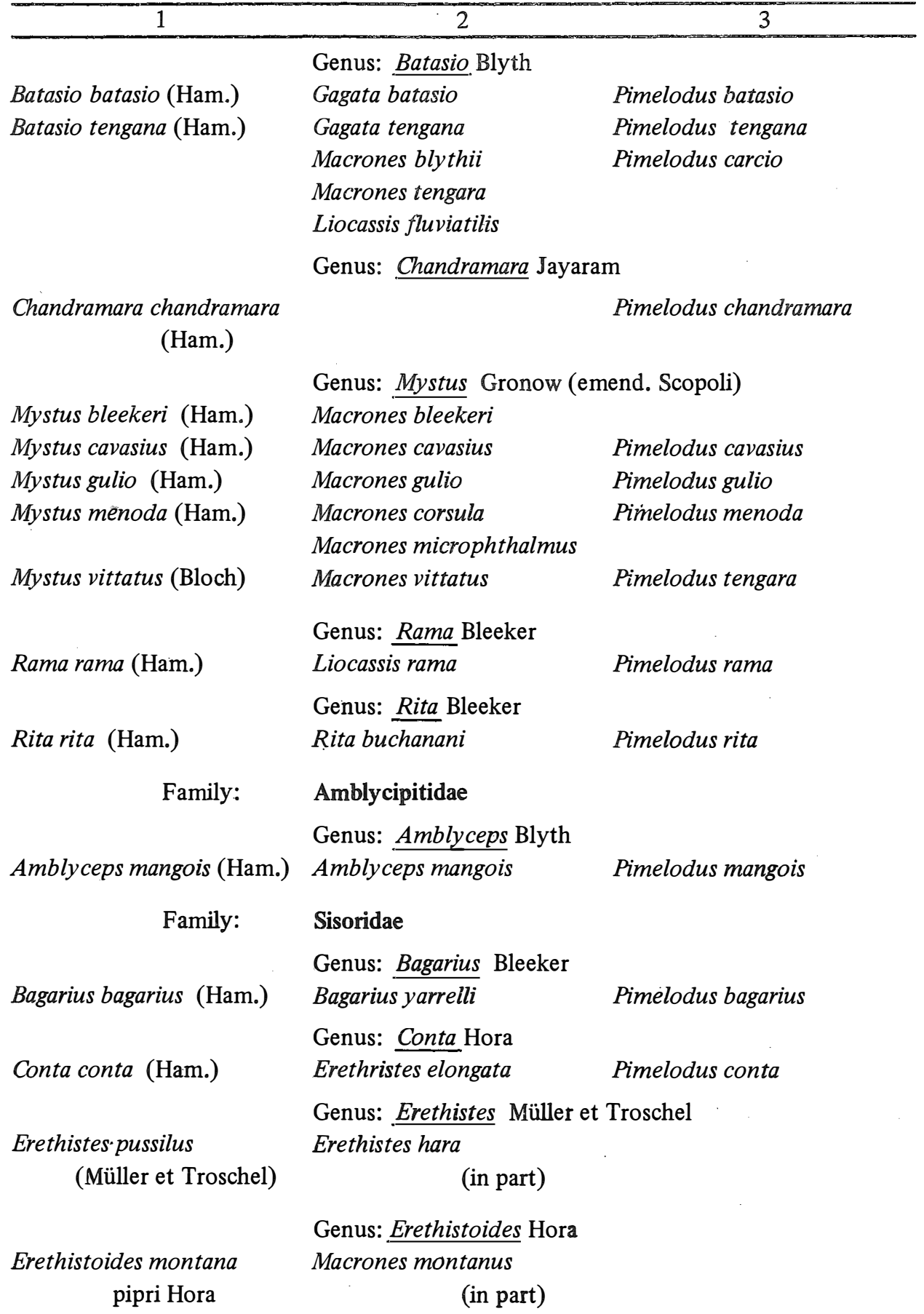


Euchliloglanis hodgarti ${ }^{12}$

Genus: Euchiloglanis Regan

Hora

Genus: Gagata Bleeker

Gagata cenia (Ham.)

Gagata gagata (Ham.)

Gagata nangra

Gagata viridescens

Gagata cenia (in part)

Pimelodus cenia

Gagata cenia (in part)

Pimelodus gagata

Nangra buchanani

Pimelodus nangra

Nangra punctata

Pimelodus viridescens

Glyptothorax annandalei

Genus: Glyptothorax Blyth

Hora

Glyptothorax cavia (Ham.) Euglyptosternum lineatum

Glyptothorax conirostris Glyptosternum conirostre

(Steind.)

Glyptosternum lonah

(in part)

Glyptothorax gracilis

Nemacheilus gracilis

(Günther)

Glyptothorax horai

Shaw et Shebbeare

Glyptothorax pectinopterus

rus (Mc Clelland)

Glyptosternum pecti-

nopterum

Glyptothorax ribeiroi

(Hora)

Glyptothorax saisi

(Jenkina)

Glyptothorax telchitta

Glyptosternum botia

Pimelicius botius

(Ham.)

Glyptosternum telchitta

Pimelocius telihitta

Genus: Hara Blyth

Hara hara (Ham.)

Erethistes hara (in part)

Pimelodus cavia

(n)




\section{1 \\ 12 \\ Genus: Pseudocheneis Blyth \\ Pseudocheneus sulcatus \\ (Mc Clelland) \\ Pseudocheneis sulcatus \\ Genus: Sisor Hamilton}

Sisor rhabdophorus Ham. Sisor rhabdophorus

Family: Chacidae

Genus: Chaca Gray

Chaca chaca (Ham.)

Ailia coila $(\mathrm{Ham} .)^{13}$

Clupisoma garua (Ham.) $)^{14}$

Chaca lophioides

Platystacus chaca

Genus: Ailia Gray

Ailia coila

Malapterus coila

Genus: Clupisoma Swainson

Pseudeutropius garua

Silurus (Callichrous) garua

Genus: Eutropiichthys Bleeker

Eutropiichthys murius

(Ham.)

Eutropiichthys vacha

(Ham.)

Pseudeutropius murius

Pimelodus murius

Eutropiichthys vacha

Pimelodus vacha

Genus: Pangasius Valenciennes

Pangasius buchanani

Pimelodus pangasius

Genus: Pseuddeutropius Bleeker

Pseudeutropius athere-
noides (Bloch) Pseudeutropius athere-

Pimelodus angius

noides

Pimelodus urua

Genus: Silonia Swainson

Silonia silondia (Ham.)

Silondia gangetica

Pimelodus silondia

Family: $\quad$ Saccobranchidae (Heteropneustidae)

Genus: Heteropneustes Müller

Heteropneustes fossilis

Saccobranchus fossilis

Silurus singio

(Bloch)

Family: Clariidae

Genus: Clarias (Scopoli)

Clarias batrachus (L.)

Clarias magur

Clarias assamensis
Macropteronotus jagur Macropteronotus magur

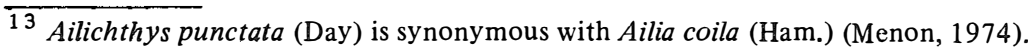

${ }^{14}$ Clupisoma montana Hora is synonymous with Eutropichthys murius (Ham.) (Menon, 1974). 


$1+23$

Order: $\quad$ ANGUILLIFORMES

Sub Order: ANGUILLOIDEI

Family: Anguillidae

Genus: Anguilla Shaw

Anguilla bengalensis

Anguilla bengalensis

Muraena anguilla

(Gray et Hardw.)

Family: Muraenidae

Genus: Muraena Artedi

$\begin{array}{lll}\text { Muraena sathete }(\mathrm{Ham} .) & \text { Muraena sathete } & \text { Muraenophis sathete } \\ \text { Muraena tile }(\mathrm{Ham} .) & \text { Muraenatile } & \text { Muraenophis tile }\end{array}$

Genus: Muraenesox Mc Clelland

Muraenesox cinereus

(Forskal)

Muraenesox cinereus

Muraena bagio

Genus: Raitaborua Gray

Raitaborua raitaborua

Moringua raitaborua

Muraena raitaborua

(Ham.)

Family: Ophichthyidae

Genus: Pisodonophis Kaup

Pisodonophis boro (Ham.) Ophichthys boro

Ophisurus boro

Ophicurus harancha

Ophisurus hijala

Order: BELONIFORMES

Sub Order: SCOMEROSOCOIDEI

Family: Belonidae

Strongylura strongylura Belone strongylura

(Van Hasselt)

Genus: Xenentodon Regan

Xenentodon cancila (Ham.) Belone cancila Esox cancila

Sub Order: EXOCOETOIDEI

Family: Hemirhamphidae

Genus: Hyporhamphus Gill

Hyporhamphus gaimardi ${ }^{15}$ Hemirhamphus limbatus

(Val.)

${ }^{15}$ Hemirhamphus gorakhpurensis Srivastava is synonymous with Hyporhamphus gaimardi (Val.) (Menon, 1974). 


\begin{tabular}{cl}
\hline 1 & \multicolumn{1}{c}{2} \\
\hline $\begin{array}{l}\text { Genus: Zenarchopterus Gill } \\
\text { Hemirhamphus ectuntio }\end{array}$ Esox ectuntio \\
(Ham.) & \\
Order: & SYNGNATHIFORMES \\
Sub Order: & SYNGNATHOIDEI \\
Family: & Syngnathidae
\end{tabular}

Genus: Doryichthys Kaup

Doryichthys councalus

Doryichthys cuncalus

Syngnathus cuncalus

(Ham.)

Doryichthys deocata

Doryichthys deocata

Syngnathus deocata

(Ham.)

Genus: Ichthyocampus Kaup

Ichthyocampus carcee

Ichthyocampus carcee

Syngnathus carcee

(Ham.)

Order: CYPRINODONTIFORMES

Sub Order: CYPRINODONTOIDEI

Family: Cyprinodontidae

Genus: Aplocheilus Mc Clelland

Aplocheilus panchax

Haplocheilus panchax

Esox panchax

(Ham.)

Oryzias melastigma

Genus: Oryzias Jordan et Snyder

Haplocheilus melanostigma

(Mc Clelland)

Order: MUGILIFORMES

Sub Order: $\quad M U G I L O I D E I$

Family: Mugilidae

Genus: Liza Jordan et Swinson

Liza parsia (Ham.)

Mugil parsia

Mugil parsia

Mugil olivaceous

Mugil jerdoni

Mugil subviridis

Mugil oligolepis

Mugil dussumieri

Liza tade (Forskal)

Mugil tade

Mugil albulla

Mugil belanak 


\section{1}

1

Mugil cephalus Linnaeus

Mugil macrolepis

(Smith) Aguas

Rhinomugil corsula (Ham.)

Sicamugil cascasia (Ham.)

Order:

Family

Eleutheronema tetradactylum Shaw

Polynemus indicus Shaw

Polynemus paradiseus L.
Genus: Mugil Linnaeus

Mugil oeur

Mugil borneensis

Mugil poicilus

Mugil troschelli

Genus: Rhinomugil Gill

Genus: Sicamugil Fowler

Mugil cascasia

Mugil cascasia

Mugil cephalus

Mugil corsula

3

POL YNEMIFORMES

Polynemidae

Genus: Eleutheronema Bleeker

Polynemus tetradactylus Polynemus teria

Genus: Polynemus Linnaeus

Polynemus indicus Polynemus sele

Polynemus paradiseus Polynemus risua

Polinemus aureus

Polyneus toposui

Order: : OPHIOCEPHALIFORMES (CHANNIFORMES)

Family: Ophiocephalidae (Channidae)

Genus: Channa Scopoli

Channa barca (Ham.)

Channa marulius (Ham.)

Channa orientalis

(Bloch et Schn.)

Channa punctatus (Bloch)

Channa stewartii

(Playfair)

Channa striatus (Bloch)
Ophiocephalus barca

Ophiocephalus marulius

Channa orientalis

Ophiocephalus gachua

Ophiocephalus punctatus

Ophiocephalus stewartii

Ophiocephalus striatus

SYMBRANCHIFORMES

Order:

SYMBRANCHOIDEI

Family:

Ophisternon bengalensis

(Mc Clelland)
Symbranchidae

Genus: Ophisternon Mc Clelland

Symbranchus bengalensis
Ophiocephalus barca

Ophiocephalus marulius

Ophiocephalus gachua

Ophiocephalus aurantiacus

Ophiocephalus lata

Ophiocephalus wrahl

Ophiocephalus chena 


\begin{tabular}{|c|c|c|}
\hline 1 & 2 & 3 \\
\hline & \multicolumn{2}{|l|}{ Genus: Monopterus Lacépède } \\
\hline \multirow{5}{*}{$\begin{array}{l}\text { Monopterus cuchia (Ham.) } \\
\qquad \begin{array}{c}\text { Order: } \\
\text { Sub Order: } \\
\text { Family: }\end{array}\end{array}$} & Amphipnous cuchia & Unibranchapertura cuchia \\
\hline & PERCIFORMES & \\
\hline & PERCOIDEI & \\
\hline & Centropomidae & \\
\hline & Genus: Chanda Fiamilton & \\
\hline \multirow{5}{*}{$\begin{array}{l}\text { Chanda baculis Ham. } \\
\text { Chanda nalua Ham. } \\
\text { Chanda nama Ham. }\end{array}$} & Ambassis baculis & Chanda baculis \\
\hline & Ambassis nalua & Chanda nalua \\
\hline & Ambassis nama & Chanda nama \\
\hline & & Chanda phula \\
\hline & & Chanda bogoda \\
\hline \multirow[t]{2}{*}{ Chanda ranga Ham. } & Ambassis ranga & Chanda ranga \\
\hline & & Chanda lala \\
\hline Family: & Serramidae & \\
\hline & Genus: Epinephelus Bloch & \\
\hline \multirow{3}{*}{$\begin{array}{l}\text { Epinephelus malabaricu: } \\
\qquad \begin{array}{r}\text { (Schn.) } \\
\text { Family: }\end{array}\end{array}$} & Serranus malabaricus & Bola (? / coioides \\
\hline & Theraponidae & \\
\hline & Genus: Therapon Cuvier & \\
\hline \multirow{3}{*}{$\begin{array}{c}\text { Therapon jarbua (Forskal) } \\
\text { Family: }\end{array}$} & Therapon jarbua & Coius trivittatus \\
\hline & Percidae & \\
\hline & Genus: Lates Cuvier et Vale & ciennes \\
\hline \multirow{2}{*}{$\begin{array}{l}\text { Lates calcarifer (Bloch) } \\
\text { Family: }\end{array}$} & Lates calcarifer & Coius vacti \\
\hline & Sillaginidae & \\
\hline \multirow{3}{*}{$\begin{array}{l}\text { Sillaginopsis panijus } \\
\qquad \begin{array}{r}\text { (Ham.) } \\
\text { Family: }\end{array}\end{array}$} & $\begin{array}{l}\text { Genus: Sillaginopsis Gill } \\
\text { Sillago panijus }\end{array}$ & Chilodipterus panijus \\
\hline & Carangidae & \\
\hline & Genus: Caranx Lacépède & \\
\hline \multirow{3}{*}{$\begin{array}{r}\text { Caranx carngus (Bloch) } \\
\text { Family: }\end{array}$} & Caranx carangus & \\
\hline & Lutianidae & \\
\hline & Genus: Lutianus Bloch & \\
\hline \multirow{3}{*}{$\begin{array}{r}\text { Lutianus johnii (Bloch) } \\
\text { Family: }\end{array}$} & Lutianus johnii & Coius catus \\
\hline & Lobotidae & \\
\hline & Genus: Datnioides Bleeker & \\
\hline
\end{tabular}




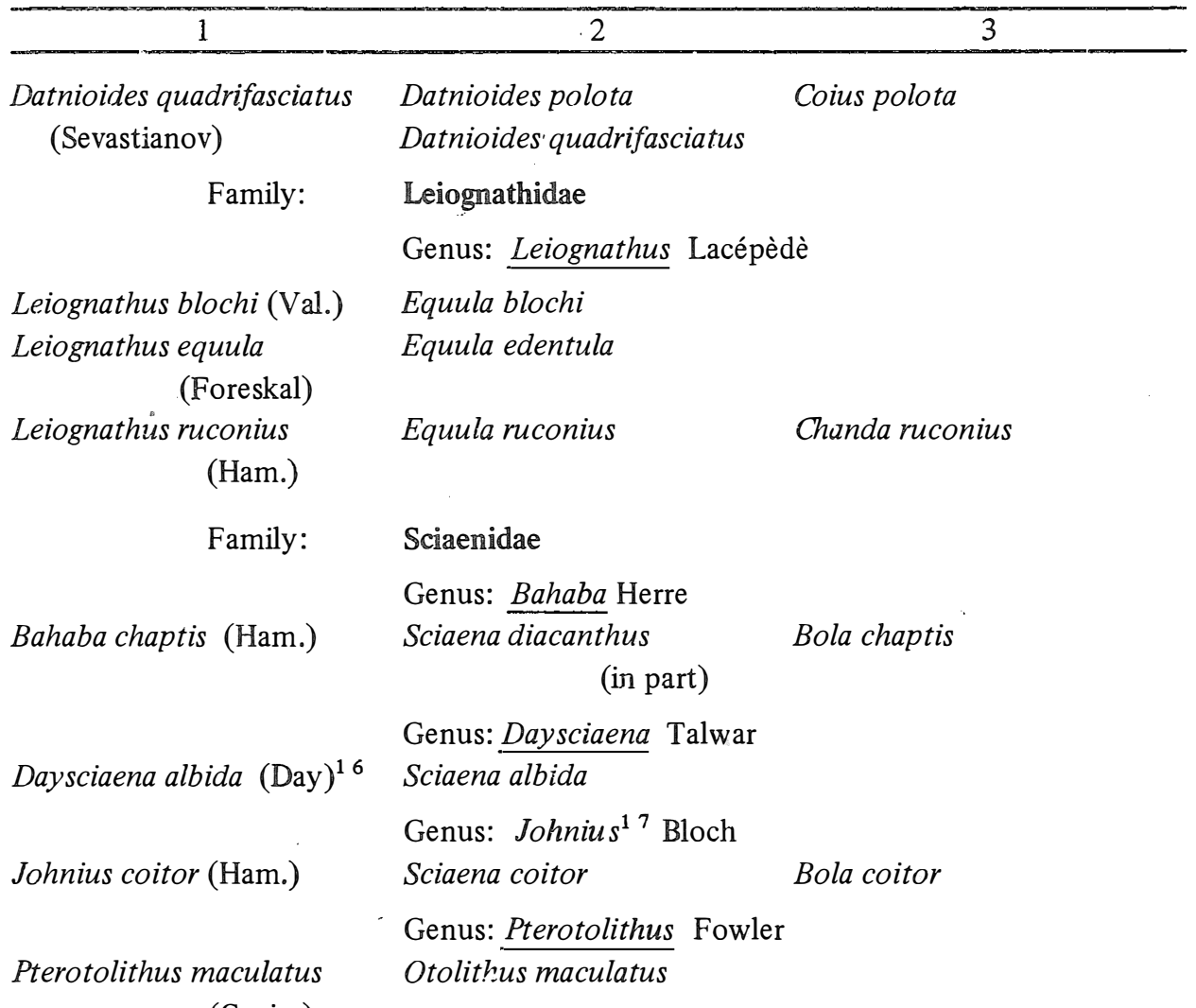

(Cuvier)

Pama pama (Ham.)

Genus: Pama Fowler

Sciaena pama

Bola pama

Genus: Macrospinosa Mohan

Macrospinosa cuja (Ham.)

Sciaena cuja

Bola cuja

Family: Toxotidae

Genus: Toxotes Cuvier

Toxotes chatareus (Ham.) Toxotes chatareus Coius charareus

Family: Sparidae

Genus: Acanthopagrus.

Acanthopagrus berda

Chrysophry's datnia

Coius datnia

(F'orskal)

Family: $\quad$ Scatophagidae

\footnotetext{
16 Dendrophysa hooglensis Sinha et Rao is synonymous with Daysciaena albida (Day) (Talwar, 19 ).

17 Genus Johnius Bioch, 1793 is synonymous with Sciaena Linnaeus, 1758 (vide: Hureau et Monod "Clofnam" I, 137).
} 


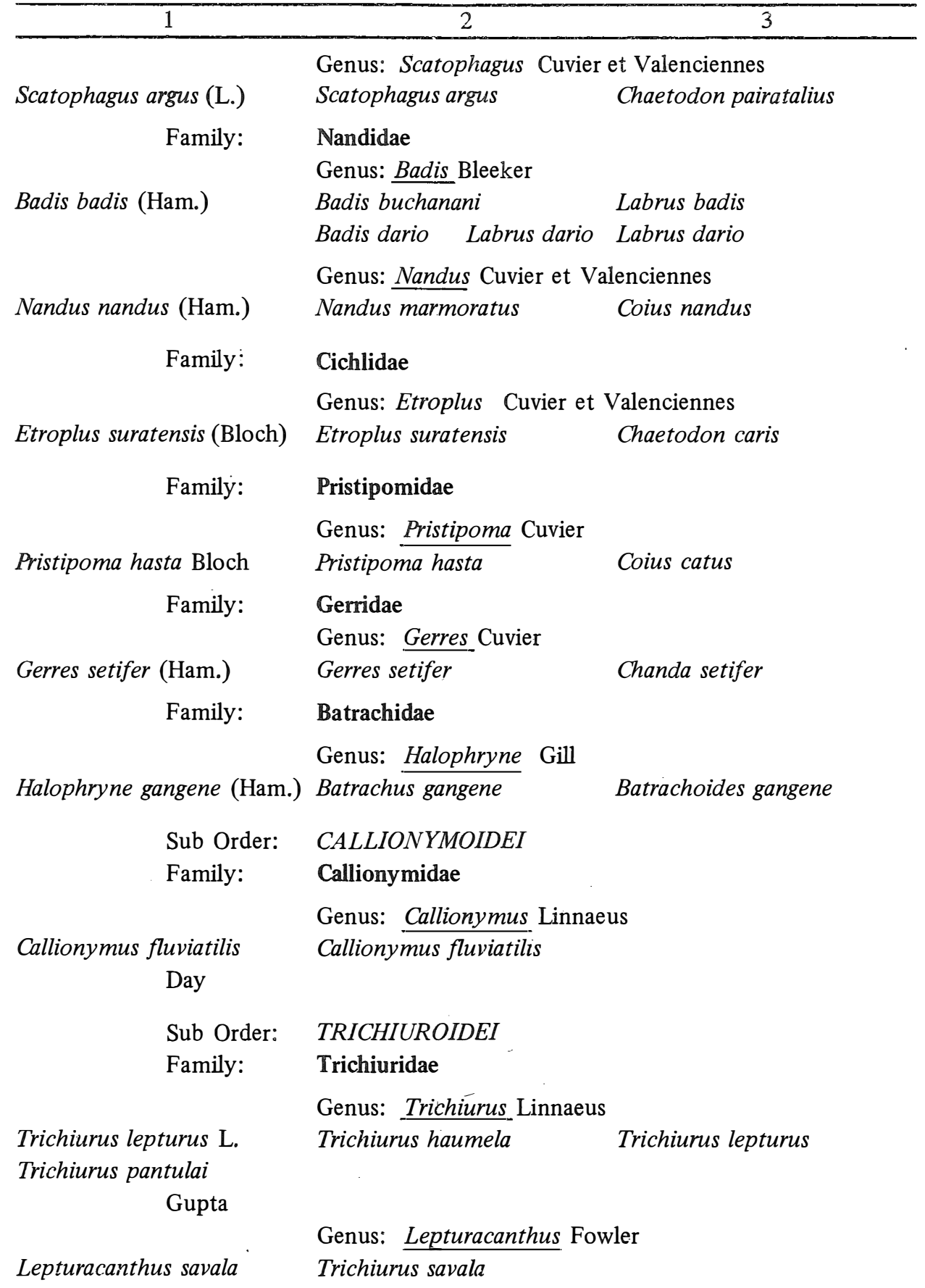

(Cuvier) 


\begin{tabular}{|c|c|c|}
\hline 1 & 2 & 3 \\
\hline Sub Order: & ANABANTOIDEI & \\
\hline Family: & Anabantidae & \\
\hline & Genus: Anabas Cuvier & \\
\hline \multirow[t]{2}{*}{$\begin{array}{l}\text { Anabas testudineus } \\
\qquad \text { (Bloch) }\end{array}$} & Anabas scandens & Coius cobojius \\
\hline & Genus: Colisa Cuvier et & lenciennes \\
\hline $\begin{array}{r}\text { Colisa fasciata (Bloch et } \\
\text { Schn.) }\end{array}$ & Trichogaster fasciatus & $\begin{array}{l}\text { Trichopodus colisa } \\
\text { Trichopodius bejeus } \\
\text { Trichopodus cotra }\end{array}$ \\
\hline Colisa labiosa (Day) & Trichogaster labiosus & \\
\hline Colisa lalia (Ham.) & Trichogaster lalius & Trichopodus lalius \\
\hline Colisa sota (Ham.) & Trichogaster chuna & Trichopodus sota \\
\hline
\end{tabular}

Sub Order: Gobioidei

Family: Gobiidae

Sub Family: Gobiinae

Genus: Brachygobius Bleeker

Brachygobius nunus (Ham.) Gobius nunus

Gobius nunus

Genus: Glossogobius Gill

Glossogobius giuris

Gobius giuris

Gobius giuris

(Ham.)

Gobius gutum

Gobius gutum

Genus: Stigmatogobius Bleeker

Stigmatogobius sadanun- Gobius sadanundio

Gobius sadanundio dio (Ham.)

Sub Family: Apocrypteinae

Genus: Apocryptes Cuvier et Valenciennes

Apocryptes bato (Ham.) Apocryptes bato Gobius bato

$\begin{array}{ccc}\begin{array}{c}\text { Pseudapocryptes lanceo- } \\ \text { latus (Bloch et Schn.) }\end{array} & \text { Apocryptes lanceolatus } & \begin{array}{c}\text { Pseudapocryptes } \\ \text { Gobio changua }\end{array}\end{array}$

Genus: Boleophthalmus Cuvier et Valenciennes

Boleophthalmus bodaerti Boleophthalmus viridis Gobius plinianus

(Pallas)

Sub Family: Periophthalminae

Genus: Periophthalmodon Bleeker

Periophthalmodon schlo- Periophthalmus schlosse- Gobius septemradiatus sseri (Pallas) ri Gobius novemradiatus 


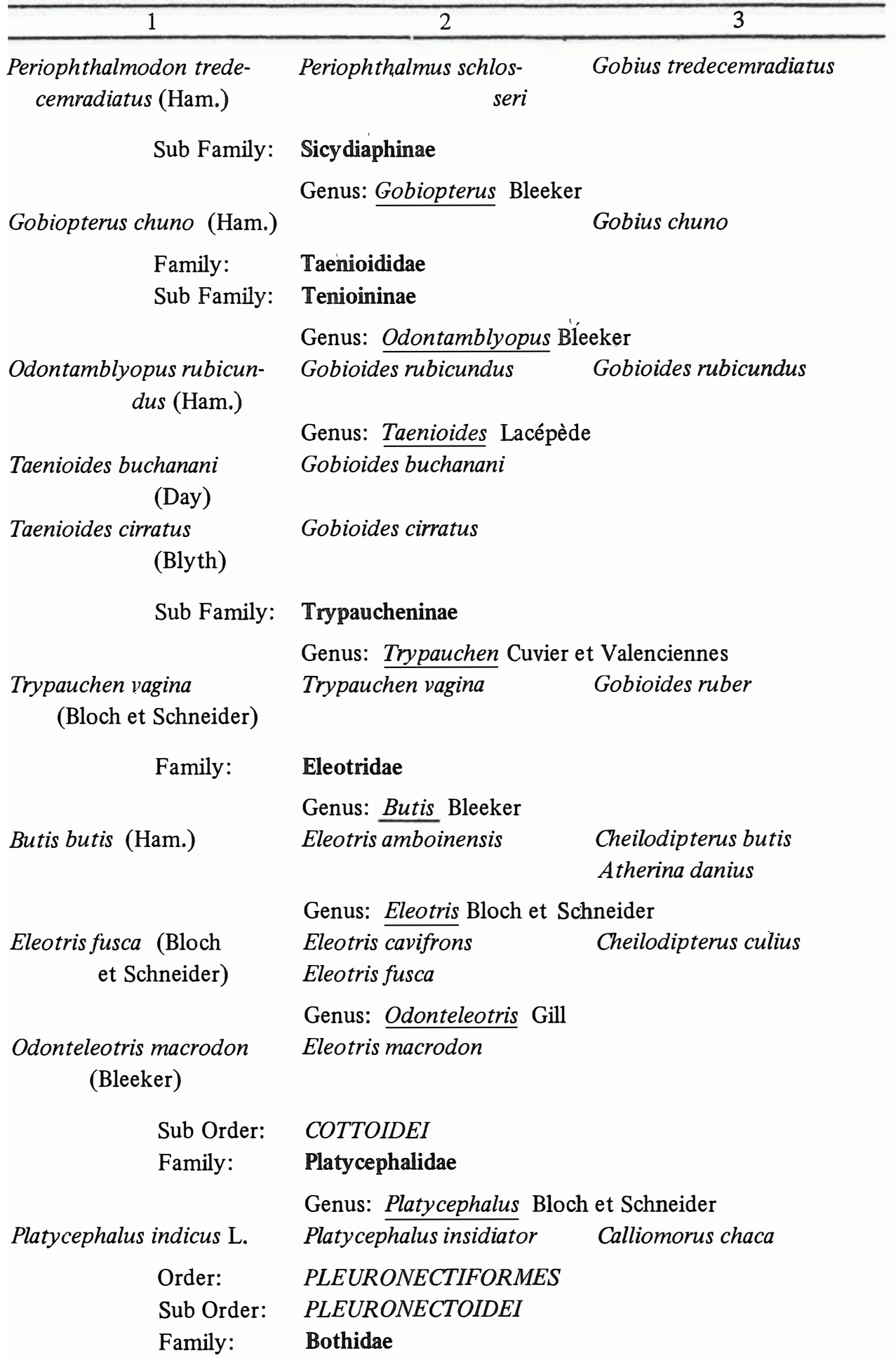


1 2

Genus: Pseudorhombus Bleeker

Pseudorhombus arsius Pseudorhombus javanicus

(Bleeker)

Family: Soleidae

Genus: Euryglossa Kaup

Euryglossa pan (Ham.)

Synaptura pan

Pleuronectes pan

Family: Cynoglossidae

Genus: Cynoglossus Hamilton

Cynoglossus cynoglossus (Ham.)

Cynoglossus lingua (Ham.)

Cynoglossus punticeps

(Richardson)

Cynoglossus bengalensis

Achirus cynoglossus

Cynoglossus lingua

Cynoglossus lingua

Cynoglossus punticeps

Cynoglossus brevis

Order:

MASTA CEMBELIFORMES

Family:

Mastacembelidae

Genus: Macrognathus Lacépède

Macrognathus aculeatus ${ }^{18}$

Rhynchobdella aculeata

Macrognathus aculeatus

(Bloch)

Genus: Mastacembelus Scopoli

Mastacembelus armatus ${ }^{19}$

(Lacépède)

Mastacembelus pancalus

(Ham.)

Order:

TETRODONTIFORMES

Sub Order: TETRODONTOIDEI

Family:

Trtrodontidae

Genus: Chelondon Müller

Chelonodon patoca (Ham.) Tetrodon patoca

Tetrodon patoca

Genus: Tetraodon Linnaeus

Tetraodon cutcutia Ham. Tetrodon cutcutia
Macrognathus armatus

Macrognathus pancalus

\section{Tetrodon cutcutia \\ Tetrodon caria \\ Tetrodon gularis}

\footnotetext{
${ }_{18}^{8}$ Rynchobdella dhanshori Hora is synonymous with Macrognathus aculeatus (Bloch) (Menon, 1974)

19 Mastacembelus manipurensis Hora is synonymous with Mastocembelus armatus (Lacépède) (Menon, 1974).
} 


$\begin{array}{lll}\text { Tetraodon fluviatilis } & \text { Tetrodon fluviatilis } & \text { Tetrodon fluviatilis } \\ \text { Tetraodon lunaris } & \text { Tetrodon lunaris } & \text { Tetrodon tepa } \\ \text { (Bloch et Schneider) } & & \end{array}$

\section{ACKNOWLEDGEMENTS}

Our thanks are due to the Director, Zoological Survey of India, Calcutta for providing facilities and to Dr. K.C. Jayaramakrishnan and Dr. P.K. Talwar of Z.S.L., Calcutta for going throgh the manuscript critically and valuable suggestions.

\section{REFERENCES}

Berg, L.S., 1940: Classification of fishes both recent and fossil. - Trav. Inst. Zool. Acad. U.S.S.R., Leningrad, 5: 87-517, 190 figs.

Chaudhuri, B.L., 1912: Descriptions of some new species of fresh water fishes from North India. Rec. Indian Mus., 7: 437-444.

Chauhan, B.S., 1947: Fish and fisheries of Patna State, Orissa. - Rec. Indian Mus., 45: 267-282.

Das, K.N., 1948: Fishes of Hazaribagh District. - Rec. Indian Mus., 41: 437-450.

David, A., 1954: A preliminary survey of the fish and Fisheries od a five mile stretch of the Hooghly river near Barrackpur.- Indian J. Fish., 1:231-255.

Day, F., 1875-1888: The fishes of India being a natural history of the fishes known to inhabit the seas and fresh waters of India, Burma and Ceylon, London: XX + 816.,198 pls.

Hora, S.L., 1921: On some new or rare species of fish from the Eastern Himalayas. - Rec. Indian Mus.,22/5/:731- 744 .

- - ., 1929: Ansid to the study of Hamilton Bauchanan's "Gangetic Fishes".- Mem. Indian Mus., 9:169-192.

- - ., 1938: On a collection of fish from the Rajmahal Hills, Santhal Paraganas, Bihar. - Rec. Indian Mus., 40/2/:169-181.

- - - ., 1948: Systematic position of Siluroides in Hamilton's "Gangetic Fishes". - Rec. Indian Mus., 47/2/:183-202.

_ - ., 1949: Siluroid fishes of India, Burma and Ceylon. XIII. Fishes of the genera Erethistes Müller and Troschel, Hara Bly th and two new allied genera. - Rec. Indian Mus., 47:183-201.

- - ., 1949: The fish fauna of the Rihand river and its Zoogeographical significance. - J. zool. Soc. India, $1: 1-7$.

- - - ., 1955: The status of Satpura Hypothesis. - Bull. nat. Inst. Sci. India, Calcutta, 7:227-229.

Hora, S.L., and Mukhejee, D.D. 1936: Fishes of the Eastern Doons, U.P. Rec. Indian Mus., 38 /2/:133-146.

Hamilton, F., 1822: An account of the fishes found in the river Ganges and its branches. Archebald Constable and Co., Edinburgh: I-VII $+1-405+39$ pls.

Jayaram, K.C., 1953: Siluroid fishes of India, Burma and Ceylon. XIV. Fishes of the Genus Mystus Scopoli. - Rec. Indian Mus., 51/4/:527-558.

- - ., 1962: Systematic status of Ailichthys punctata Day and its relationship with Ailia coila (Hamilton), (Siluridea: Schilbeidae). - J. zool. Soc. India., 14/2/:244-248.

Jhingran, V.G., 1956: The capture fishery of river Ganga at Buxar (Bihar, India) in the years 1952-1954. -- Indian J. Fish., 4/1:197-215. 
Karamchandani, S.Z., 1962: On a collection of fishes from the Ganges river at Dighwara (Bihar). Tropical Ecol., 3/1,2/:79-83.

Lal, M.B. and Chatterjee, P., 1962: Survey of Eastern Doon fishes with certain notes on their Biology. - J.zool. Soc. India., 14/2/:230-243.

Mahajan, C.L., 1965: Fish fauna of Muzaffarnagar District, Uttat Pradesh. - J. Bombay Nat. Hist. Soc., $62 / 3 /: 440-462$.

Majumdar, N.N. 1978: On a collection of fish from Deihi State. - J. Bombay Nat. Hist. Soc., $55 / 2 /: 366-370$.

Menon, A.G.K., 1949: Notes on fishes in the Indian Museum. XLIV. Fishes From the Kosi Himalayas, Nepal. - Rec. Indian Mus., 47/2/:231-237.

- - - ., 1949: Fishes of the Kumaon Himalayas. - J. Bombay Nat. Hist. Soc., 48/3/:5 35-542.

- - ., 1962: A distributional list of fishes of the Himalayas. - J. zool. Soc. India., 14/1,2/:23-32.

- - ., 1971: Taxonomy of fishes of the Genus Schizothorax Heckel with the description of a new species from Kumaon Himalayas - Rec. zool. Surv. India., 63/1-4/:195-207.

-- - ., 1974: A check list ot Fishes of Himalay an and the Indo-Gangetic Plains. I.F.S.I., Barrackpur: I-VIII, 1-136.

Misra, K.S., 1959: An aid to the identification of the common commercial fishes of India and Pakistan. - Rec. Indian Mus., 57/1-4/:1-320.

Motwani, M.P. and David, A., 1957: Fishes of the river Sone with observations of the Zoogeographical significance. - J. zool. Soc. India., 9/1/:9-14.

Pant, M.C., 1966: Fish fauna of the Kumaon hills. - Rec. zool. Surv. India, 64/1-4/:85-96.

Shaw, G.E. and Shebbeare, E.O., 1938: The fishes of Northern Bengal. - J. Roy. Asiat. Soc. Bengal (Science), 3:1-137, 8 pls.

Sinha, B.M. and Shiromony, P.A., 1953: The fish of Meerut. - Rec. Indian Mus., 51/1/:61-65.

Srivastava, G.J., 1967: A new species of Fresh water fish of the Genus Hemirhamphus Cuv. from Gorakhpur, Uttar Pradesh, India. - J. Bombay Nat. Hist. Soc., 64/1/:93-94.

- - ., 1968: Fishes of Eastern Uttar Pradesh. Vishwavidyalaya Prakashan, Varanasi: I-XXII, $1-163$.

Venkateswarlu, T., 1972: Fauna of Bihar State (India). 1. Fishes. - Ind. J. Zoot. 13/3/:119-128.

- - . 1973: Scientific and local names of commercial fishes of river Ganges and its branches. - Sea Food Expt. J., 5:7-13.

- - ., 1976: On a collection of fishes from Patna District (Bihar, India). - Acta Ichthyologica et Piscatoria, 6/1/:83-107.

- - ., 1977: Fishes of river Poonpun (Bihar, India). Acta Ichthyologica et Piscatoria, 7:1/:41-58.

\section{WYKAZ RYB RZEKI GANGES I JEJ DOPŁYWÓW}

\section{Streszczenie}

Od czasu pierwszego spisu ryb Gangesu przez Hamiltona (1822) powstało wiele publikacji w latach późniejszych, wiele nazw uległo zasadniczej zmianie w nowoczesnej systematyce. W roku 1929 Hora w pracy "Gangetic Fishes" podał spis 272 gatunków, z których tylko 264 weszło do obecnego, 8 gatunków, wymienionych we wstępie, trudno obecnie zidentyfikować. Autorzy podają spis 264 gatunków opierając się na systemie Berga (1940). Jednocześnie uwzględniają synonimy podane w pracy Day'a (1875-1878) z "Fishes of India" i Hamiltona z "Gangetic Fishes". 
T. Венкатесварлу, А.Г.й. Менон

ПЕРЕЧЕНЬ РЫБ РЕКИ ГАНГ И ЕЁ ПРИТОКОВ

Реэюме

Со времени составления первого списка видов рыб Ганга Хемильтоном (Наmilton, 1822) появилось позже много статей. Многие названия видов рыб подверглись основным изменениям в современной систематике. В 1929 году Xopa (Hora) в работе "Gangetic Fishes" представил описание 272 видов, из которых только 264 вида вошли в состав настоящего списка. 8 видов, представленных во вступлении, сейчас трудно идентифицировать. Авторы дают перечень 264 видов, опираясь на систему Берга (1940). Одновременно учитывают синонимы, данные в работе Дейа (Day, 1875-1878) из "Fishes of India” и Хемильтона из "Gangetic Fishes".

Address:

Received: 10 X $1978 \mathrm{r}$.

T. Venkateswarlu - Zoologist

Southern Regional Station

Zoological Survey of India

100 San thome High Road

Madras - 600028, India 